\section{Organic dust in comet Halley}

SIR-In a recent article by Kissel and Krueger ${ }^{1}$ a statement is made to the effect that the hypothesis of biological grains has been disproved for the organic dust from comet Halley as analysed by Vega 1 . This conclusion turns out to be contingent on several assumptions that are artificially introduced into the data analysis. First, the implicit assumption that all of the cometary dust in our model ${ }^{2}$ is biological may be incorrect, for even under optimal laboratory conditions a 20 per cent conversion of nutrient into cells would be considered an excellent yield. Second, cometary dust particles colliding with the mass spectrometer at speeds of $70 \mathrm{~km} \mathrm{~s}^{-1}$, corresponding to an energy of $300 \mathrm{eV}$ per carbon atom, could surely not be observed ionization, dissociation and subsequent recombinations would have the effect of considerably modifying initial chemical structures. Attempts to reconstruct theoretical considerations involve uncertainties to a major degree. Furthermore, the differing distributions of ionization states for break-up ions corresponding to such elements as $\mathrm{P}, \mathrm{Na}$ and $\mathrm{K}$ cannot be determined to any degree of precision, thus leading to uncertainties in estimates of elemental ratios. Finally, any estimate of the distribution of elements between the presumptive 'chondritic core' and a mantle is an artefact of modelling not necessarily connected with the properties of cometary dust. Model-dependent discriminatory criteria such as these cannot be used in logic as disproof of any particular model.

Perhaps more significantly the only data relating to pristine grains from comet Halley were derived not from expensive satellite experimentation but using ground-based telescopes such as the in anything like their pristine state. Impact original molecular configurations using

Anglo-Australian Telescope. The figure shows the infrared data for the dust from this comet observed on 30 March 1976 compared with the predictions of the bacterial model ${ }^{3}$.

N.C. WICKRAMASINGHE Department of Applied Mathematics and Astronomy, Cathays Park, PO Box 78, Cardiff CF1 $1 X L, U K$

1. Kissel, J. \& Krueger, F.R. Nature 326, 755-N. (1987) . Hoyle, F. \& Wickramasinghe, N.C. Living Comets (University College Press, 1985)

3. Wickramasinghe, D.T. \& Allen, D.A. Nature 323-- (1986)

KisSEl and KRUEGER REPLY - We would like to make some remarks concerning our article on the organic component in the dust from comet Halley. As we have already communicated elsewhere ${ }^{2}$, the different distribution states of ionization of the atomic ions (such as $\mathrm{Na}^{+}, \mathrm{K}^{+}, \mathrm{P}^{+}$) after impact of a dust particle are well known within a factor of two over the periodic table. With these ion/atom yield ratios one recognizes the mineral parts of the grains as being, on average, chondritic, and the organic parts not containing phosphorous and with only $\sim 10^{-6}$ sodium and potassium, with lines that may even be due to target contaminations only, as we have recently confirmed. Together with the coma gas measurements ${ }^{3}$, the total elemental distribution (of gas, mineral and organic dust) fits the solar distributions ${ }^{4}$ well; from this we are even more convinced about the validity of our atomic ionization distribution. But we admit that this was not the scope of the paper cited by Hoyle and Wickramasinghe; therefore we have decided to write a more comprehensive article ${ }^{5}$ about the methods and results of the elemental and anorganic composition.

Freeze-dried cells should contain at least a percentage of sodium and 0.1 per cent of potassium, which is clearly not the

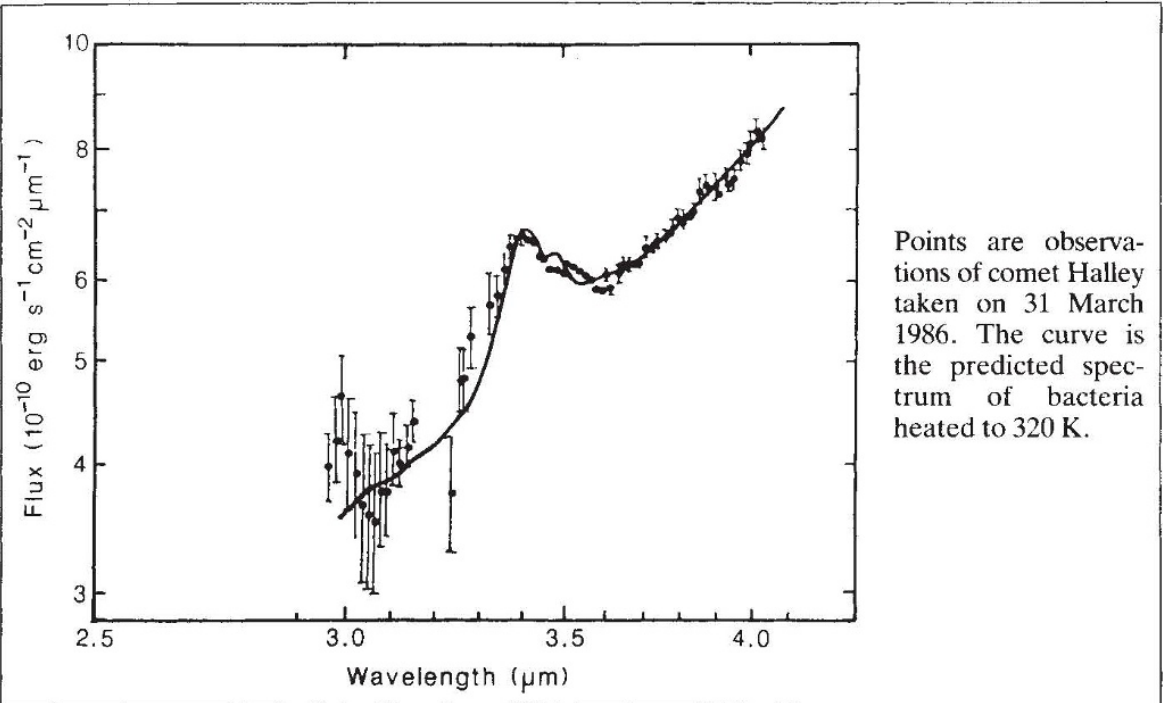

case with the organic grains. Even the anorganic mineral parts contain less than $3 \times 10^{-3} \mathrm{Na}$, less than $1 \times 10^{-4} \mathrm{~K}$, and less than $5 \times 10^{-4} \mathrm{P}$. The organic part contains less than $10^{-5} \mathrm{P}$, which is not sufficient to form sufficient nucleic acids and phospholipids.

We never claimed that the large molecules possibly present in the organic part of the dust survive the impact process; however, those organic fragment ions that show up in the mass spectra tell us about the nature of the main chemical classes being present. As we find highly reactive states apparently with hydrogen and water driven out of the molecules, these do not fit the requirements of living cells, however, in warm water the prerequisites of self-replicative units may form, as known from Orgel, Lohrmann, and co-workers ${ }^{6}$.

We are, indeed, very astonished that one may draw conclusions of living matter being present merely from infrared spectra. Instead, we feel this to be a contingent way of treating empirical data. However, more detailed interrelationships between grain size, composition, and structure with infrared and mass spectra are known today ${ }^{4}$.

Finally, we regard the disproval of the concept of 'evolution' to be even more difficult after the analysis of the cometary dust by space-borne instruments.

J. KISSEL

F.R. KRUEGER

\section{Max-Planck Institut}

für Kernphysik,

\section{D-6900 Heidelberg 1, FRG}

1. Kissel, J. \& Krueger, F.R. Nature 326, 755 (1987)

2. Jessberger, E.K Kissel, J. Fechtig, H \& Krueger, F R in Physical Processes in Comets, Stars and Active Galaxies (eds Hillebrandt, W. et al.) 26 (Springer, Berlin, 1987).

3. Krankowsky, D. et al. Nature 321, 326 (1986).

4. Greenberg, J.M. et al. Proc. Workshop on Fluffy Structures in Leiden, Netherlands, May, 1987 (submitted).

5. Krueger, F.R. \& Kissel, J. (manuscript in preparation)

6. Lohrmann, R., Bridson, P.K. \& Orgel, L.E. Science 208. 1464 (1980).

\section{Body size, energy use and ecological dominance}

SIR-Brown and Maurer ${ }^{1}$ recently claimed that large species were energetically more important in communities than small species, in contrast with earlier findings ${ }^{2,3}$. Their evidence is not compelling. Only 5 out of 39 of the bird and none of the fish regressions are statistically significant. Significance is incorrectly claimed for the mammal slope $(r=0.56$, $n=9$, therefore $P>0.1$ ); only the plant assemblages are consistently statistically significant. More seriously there is a strong correlation between the slopes of their regression lines and the corresponding correlation coefficients (birds: $r=$ $-0.76, P<0.001)$. The estimated slope for a perfect correlation is $-1.34(95 \%$ limits are between -1.07 and -1.61 ), strong evidence for the greater im- 